\title{
ANÁLISE DO COMPORTAMENTO ESTRUTURAL DE ELEMENTOS CÔNICOS DE POLIÉSTER REFORÇADO COM FIBRA DE VIDRO (PRFV)
}

\section{RESUMO}

Os materiais compósitos, apesar de muito antigos, passaram a ganhar importância no século XX, com o surgimento das fibras sintéticas. Atualmente está presente nos segmentos naval e aeronáutico, pois apresentou diversas vantagens em relação aos materiais antes utilizados como matéria-prima. Sua participação em outros segmentos do mercado ainda é limitada. O setor de distribuição de energia elétrica encontrou no material compósito uma ótima solução para postes e acessórios que precisam ser instalados em locais de difícil acesso. Porém, por existirem poucas aplicações deste material em postes de distribuição no mercado, a defasagem de informações e estudos sobre ele é grande. O presente trabalho analisa estruturalmente um poste de material compósito, mais precisamente poliéster reforçado com fibra de vidro. Foram obtidos valores de tensões e deslocamentos através de modelagem estrutural por elementos finitos, comparando os resultados da modelagem com resultados experimentais, verificando a viabilidade da modelagem para a análise até carregamentos equivalentes à resistência nominal do poste.

Palavras-Chave: Compósitos; PRFV; Poste de fibra. 
Apesar de bastante estudado nos últimos 50 anos, ainda há muito a ser pesquisado sobre as propriedades dos compósitos para viabilizá-los como um concorrente direto na indústria da construção civil. A dificuldade de se encontrar informações disponíveis sobre as propriedades e o comportamento dos compósitos se dá por dois motivos: o primeiro é ser uma tecnologia recente, quando comparado a outros materiais.
O segundo é a dificuldade da generalização de informações, já que as propriedades mudam conforme o tipo de compósito analisado e das múltiplas combinações possíveis. Com o intuito de contribuir com a disseminação de informações sobre compósitos, o presente trabalho tem por objetivo analisar estruturalmente um elemento cônico de compósito de poliéster reforçado com fibra de vidro, produzido pelo método de filamento contínuo.

\section{REVISÃO BIBLIOGRÁFICA}

\subsection{FIBRAS}

As fibras, também conhecidas como reforços do material compósito, são responsáveis pela rigidez e resistência mecânica do compósito. Podem ser naturais ou sintéticas. Dentre as fibras sintéticas, destacam-se as de aramida, vidro e carbono (FARIA, 2007). A escolha do tipo de fibra a ser utilizada é feita baseada em várias considerações: carregamentos aos quais a estrutura estará submetida, custo do material, técnica de fabricação empregada e restrições de aplicações (MARINUCCI, 2011).

As fibras sintéticas em geral apresentam elevada resistência mecânica e baixo peso específico. As fibras de vidro são as que proporcionam a melhor relação custo-benefício dentre os reforços utilizados na fabricação de compósitos poliméricos. Parte disso se deve à facilidade de encontrar as matérias-primas necessárias para a sua fabricação (MARINUCCI, 2011). Zurtrassen (2008 apud ABMACO, 2009) divide as fibras de vidro em seis tipos:
- Vidro - E (electrical glass): boa resistência a descargas elétricas (não é condutor de corrente).

- Vidro-C (chemical glass): elevada resistência química, tanto em meios ácidos como alcalinos. Normalmente utilizado na forma de véu como barreira protetora.

- Vidro-ECR: resistência química a meios ácidos.

- Vidro ecológico: não emprega boro e flúor na sua composição.

- Vidro - AR (Alkali-resistent glass): resistência química a meios básicos.

- Vidro - R ou S: são vidros de alto desempenho, apresentam elevadas propriedades mecânicas.

- Vidro - A: "High-Alkali-glass content" pouco utilizado.

- Vidro - D: Reforço de materiais eletrônicos para telecomunicação radares civis e militares 
Os tipos mais utilizados na produção de compósitos são os vidros $\mathrm{E}$ e $\mathrm{AR}$, compatíveis com vários tipos de aplicação. Os vidros de alto desempenho são utilizados na indústria aeroespacial e o tipo $\mathrm{C}$ é pouco competitivo, devido ao seu alto preço (ORTENZI Jr., 2007). Callister (2002) considera que o tamanho e o direcionamento da fibra são fatores determinantes no desempenho estrutural do compósito.

\subsection{MATRIZES}

A principal função da matriz em um material composto é servir como material ligante (RIBEIRO, 2000 apud FARIA, 2007). Além disso, Faria (2007) explica que a matriz é responsável por transferir as cargas aplicadas ao compósito para as fibras, protegendo-as.

Segundo Callister (2002), a caracterização dos polímeros utilizados para as matrizes se dá da mesma forma que a dos metais, sendo as principais propriedades: módulo de elasticidade, limite de resistência à tração e resistências ao impacto e à fadiga.

Nas resinas poliéster, é importante levar em consideração duas características fundamentais para a sua aplicação: a viscosidade e a tixotropia. A primeira determina a facilidade de impregnação das fibras de vidro, e a segunda, a capacidade de não escorrimento quando aplicada em superfícies verticais ou inclinadas (FORTE, 2014).

\subsection{POLIÉSTER REFORÇADO COM FIBRA DE VIDRO (PRFV)}

A combinação da resina poliéster com fibra de vidro resulta em um material compósito conhecido como Poliéster Reforçado com Fibra de Vidro (PRFV). O PRFV tem alta resistência à tração, flexão e impacto, e não conduz corrente elétrica. Por estes motivos, é utilizado em aplicações estruturais, principalmente nas que exigem matéria-prima isolante elétrica (FERREIRA, 2009). Além disso, a resina poliéster, se combinada com diferentes tipos de polímeros, possui boa resistência química quando exposta a meios corrosivos (CALLISTER, 2002). O autor aponta duas características importantes a serem observadas, pois podem tornar este tipo de compósito inviável para alguns tipos de aplicação. A primeira é a sua baixa rigidez, apesar de alta resistência mecânica. A segunda se deve ao fato de que sua resistência ao calor é consideravelmente baixa. Acima de $200^{\circ} \mathrm{C}$, o compósito deixa de cumprir satisfatoriamente suas funções estruturais, pois o polímero começa a se deteriorar.

\subsection{MODELOS} MATEMÁTICOS PARA ANÁLISE DE COMPÓSITOS

Diversos modelos matemáticos podem ser utilizados para a análise de materiais compósitos. A seguir, serão apresentados alguns deles.

\subsubsection{Método dos Elementos Finitos (MEF)}

Este método tem por objetivo determinar o estado de tensões e as deformações sofridas pela estrutura estudada (AZEVEDO, 2003). O MEF consiste na discretização do sistema, ou seja, dividi-lo em vários subdomínios. Quanto maior for a divisão do domínio, mais aproximado da realidade será o resultado obtido (ALVES, 2007). 
Gesualdo (2010) apresenta a equação para utilização do método:

$$
[\mathrm{K}]\{\mathrm{u}\}=\{\mathrm{F}\}
$$

Onde:

[K] é a matriz de rigidez global da estrutura; $\{\mathbf{u}\}$ é o vetor de incógnitas;

$\{\mathbf{F}\}$ é o vetor de forças.

A incógnita representada por $\{\mathbf{u}\}$ pode ser temperatura, deslocamento, velocidade, entre outros. A matriz de rigidez global $[\mathbf{K}]$ pode ser obtida através do acoplamento de todas as matrizes de rigidez locais (GESUALDO, 2010).

\subsubsection{Modelo Desenvolvido pelo National Physical Laboratory United Kingdom (NPL-UK )}

O modelo matemático desenvolvido pelo NPLUK (1999) tem como base vários outros sistemas de dimensionamento (ORTENZI Jr., 2007). Nele é proposto o dimensionamento das estruturas pela teoria dos estados limites. As equações 2 e 3 são utilizadas para o dimensionamento:

$$
\begin{gathered}
\mathrm{S}\left(\mathrm{X}_{\mathrm{LiYFLi})} \mathrm{Y}_{\mathrm{F} 3}<\mathrm{R}\left(\mathrm{X}_{\mathrm{Gi}}, \mathrm{X}_{\mathrm{Mi}} / \mathrm{Y}_{\mathrm{Mi}}\right)\right. \\
\mathrm{Y}_{\mathrm{m}}=\mathrm{Y}_{\mathrm{v}}+\mathrm{Y}_{\mathrm{E}}+\mathrm{Y}_{\mathrm{t}}
\end{gathered}
$$

Onde:

$S$ é o conjunto de solicitações, o qual deve ser menor do que $\boldsymbol{R}$, que representa a capacidade resistente da estrutura;

$\mathrm{X}_{\mathrm{Li}}$ representaa soma das ações de carregamento; $\mathbf{Y}_{\mathrm{FLi}}$ representa os fatores de segurança parciais associados;
$\mathbf{Y}_{\mathrm{F} 3}$ é o somatório dos fatores de segurança parciais de cada eixo principal;

$\mathbf{X}_{\mathrm{Mi}}$ representa a resistência dos materiais (fibra e matriz);

$\mathbf{X}_{\mathrm{Gi}}$ representa a geometria da estrutura;

$\mathbf{Y}_{\mathrm{Mi}}$ é o coeficiente de segurança dos materiais;

$\mathbf{Y}_{\mathrm{m}}$ é o fator de segurança parcial dos materiais;

$\mathbf{Y}_{\mathbf{v}}$ é o fator de probabilidade de falha por variabilidade do material;

$\mathbf{Y}_{\mathbf{E}}$ éo fator de efeitos higrotérmicos que afetam o material;

$\mathbf{T}_{\mathbf{t}}$ é o fator de probabilidade de falha por fluência e idade do material.

Este método de dimensionamento necessita de várias características do material e da estrutura, que devem ser determinadas e/ou pré-calculadas pelo projetista (ORTENZI Jr., 2007).

\subsubsection{Modelos de Análise de Falha por Ruptura}

Segundo Ortenzi (2007), para análise de falha por ruptura, são utilizados principalmente dois modelos matemáticos: o modelo de Tsai-Hill e o modelo de Tsai-Wu.

O modelo de Tsai-Hill trata de uma adaptação de dois modelos: o de Von Misses e o de Hill. Este modelo propõe a análise de uma lâmina ortotrópica no estado plano de tensões, no qual a direção principal é a do sentido longitudinal das fibras. Nas resistências de escoamento de Hill, são consideradas as resistências de escoamento da lâmina. A equação 4 rege o modelo de Tsai-Hill:

$$
\frac{\sigma 1^{2}}{S^{2}}-\frac{\sigma 1 \quad \sigma 2}{S_{L}^{2}}-\frac{\sigma 2^{2}}{S_{T}^{2}}-\frac{\tau 12^{2}}{S_{L T}^{2}}=1
$$


Onde:

$\boldsymbol{\sigma}_{1}, \boldsymbol{\sigma}_{2}$ são as tensões atuantes nas direções x e $z$, respectivamente;

$\mathbf{T}_{12}$ é a tensão de cisalhamento referente a xz;

$\mathrm{S}_{\mathrm{L}}, \mathrm{S}_{\mathrm{T}}$ e $\mathrm{S}_{\mathrm{LT}}$ são as resistências características longitudinal, transversal e de cisalhamento, obtidas por ensaios (ORTENZI Jr., 2007).
O mesmo autor ainda descreve o segundo modelo de análise por falha e ruptura, o modelo de Tsai-Wu. Diferentemente do modelo anteriormente citado, o modelo desenvolvido por Tsai e $W u$ prevê a influência das diversas camadas de lâminas e diferentes direções em relação à linha neutra. $\mathrm{O}$ equacionamento deste modelo é mais complexo do que o de Tsai-Hill, e pode ser observado na equação 5 :

$$
\sigma_{x}\left(\frac{1}{X_{t}}+\frac{1}{X_{c}}\right)+\sigma_{y}\left(\frac{1}{Y_{t}}+\frac{1}{Y_{c}}\right)+\frac{\sigma x^{2}}{\left(X_{t} X_{c}\right)}+\frac{\sigma y^{2}}{\left(Y_{t} Y_{c}\right)}+\frac{\tau x z^{2}}{S^{2}}+F_{x y} \sigma_{x} \sigma_{y}=1
$$

Onde:

$\mathbf{X}_{\mathbf{t}}$ é o tensor de tração em relação a x;

$\mathbf{X}_{\mathbf{c}}$ é o tensor de compressão em relação a x;

$\mathbf{Y}_{\mathbf{t}}$ é o tensor de tração em relação a y;

$\mathbf{Y}_{\mathbf{c}}$ é o tensor de compressão em relação a y;

$\mathbf{F}_{\mathrm{xy}}$ é o tensor somatório das resistências características biaxiais em x e y, obtido através de ensaios de tração biaxial em que $\sigma_{x}=\sigma_{y}$.

\section{PROCEDIMENTOS METODOLÓGICOS}

A seguir, serão descritos os materiais e os métodos utilizados na pesquisa, tais como características e modelagem da estrutura e ensaios realizados.

\subsection{CARACTERIZAÇÃO DO MATERIAL}

As matérias-primas utilizadas na fabricação do compósito são:

- Resina poliéster ortoftálica;
- Fibra de vidro contínua do tipo E TEX 2200 ;

- Promotor de cura peróxido de metil etil cetona;

- Pintura externa de proteção com tinta gel coat ortoftálico $7040 \mathrm{PA}+\mathrm{UV}$.

O compósito foi fabricado com uma relação de fibra/resina de 70/30\%. Além disso, foi utilizado o promotor de cura com a proporção de 1,5\% da massa de resina. 
Amostras de material equivalente foram submetidas a ensaios mecânicos em laboratório certificado pela fabricante da estrutura. Os ensaios, as normas de referência e os resultados estão expostos no Quadro 1:

Quadro 1: Ensaios de caracterização de PRFV

\begin{tabular}{|c|c|c|c|c|c|}
\hline \multirow[b]{2}{*}{ Ensaio } & \multirow{2}{*}{$\begin{array}{l}\text { Norma de } \\
\text { referência }\end{array}$} & \multicolumn{2}{|c|}{ Tensão média de ruptura (MPa) } & \multicolumn{2}{|c|}{ Alongamento médio (\%) } \\
\hline & & $\begin{array}{c}\text { Antes do } \\
\text { envelhecimento }\end{array}$ & $\begin{array}{c}\text { Após o } \\
\text { envelhecimento }\end{array}$ & $\begin{array}{c}\text { Antes do } \\
\text { envelhecimento }\end{array}$ & $\begin{array}{c}\text { Após o } \\
\text { envelhecimento }\end{array}$ \\
\hline $\begin{array}{l}\text { Resistência à } \\
\text { tração }\end{array}$ & $\begin{array}{l}\text { ASTM } \\
\text { D638 }\end{array}$ & 321,6 & 355,7 & 4,0 & 4,0 \\
\hline $\begin{array}{l}\text { Resistência } \\
\text { à flexão }\end{array}$ & $\begin{array}{c}\text { ASTM } \\
\text { D790 }\end{array}$ & 327,0 & 389,0 & - & - \\
\hline
\end{tabular}

Fonte: Adaptado de TECHNOFIX (2016)

O coeficiente de Poisson do material é 0,32 e o módulo de elasticidade do material é 27,7 GPa, valores obtidos da Technofix Ind. e Com. de fibras de vidro.

\subsection{CARACTERÍSTICAS DA ESTRUTURA}

A estrutura estudada neste trabalho é a de um poste para distribuição de energia. Suas dimensões estão apresentadas na Tabela 1 :

Tabela 1: Dimensões da estrutura

\begin{tabular}{l|c}
\hline Diâmetro do topo $(\mathrm{mm})$ & 165 \\
\hline Diâmetro da base $(\mathrm{mm})$ & 370 \\
\hline Comprimento total $(\mathrm{mm})$ & 12000 \\
\hline Resistência nominal $(\mathrm{daN})$ & 200 \\
\hline Espessura da parede $(\mathrm{mm})$ & 5 \\
\hline
\end{tabular}

Fonte: TECHNOFIX (2016)

A resistência nominal da estrutura é a força à qual a estrutura estará submetida constantemente quando instalada. Como no Brasil ainda não existe norma regulamentadora para postes
dePRFV, foram seguidas as diretrizes da norma ABNT NBR 8451 (ABNT, 2011) - Postes de concreto armado para redes de distribuição de energia elétrica. Esta norma estabelece que o poste suporte uma carga duas vezes maior que 
sua resistência nominal e que o deslocamento do topo seja limitado pela concessionária de energia elétrica do estado onde o poste será instalado, que no presente estudo corresponde a $4 \%$ do comprimento total do poste.

A espessura de $5 \mathrm{~mm}$ foi composta por camadas de fibra impregnada por resina, enroladas com ângulo de aproximadamente 23 graus. Apenas nos dois primeiros metros, a contar do ponto de engastamento, as duas primeiras e duas últimas camadas de fibra foram enroladas com ângulo de 90 graus, a fim de evitar o fenômeno de ovalização do poste.

\subsection{ENSAIO DE CAMPO DA ESTRUTURA}

Foi produzido um poste em escala real para fazer a análise do deslocamento do todo e da variação do diâmetro na região próxima ao engastamento, quando solicitada às cargas de trabalho aplicada a $10 \mathrm{~cm}$ do topo. Esta metodologia de ensaio foi selecionada com base no método de ensaio proposto pela NBR 8451 (ABNT,2011). Na Figura 1, pode-se observar a estrutura submetida à força citada.

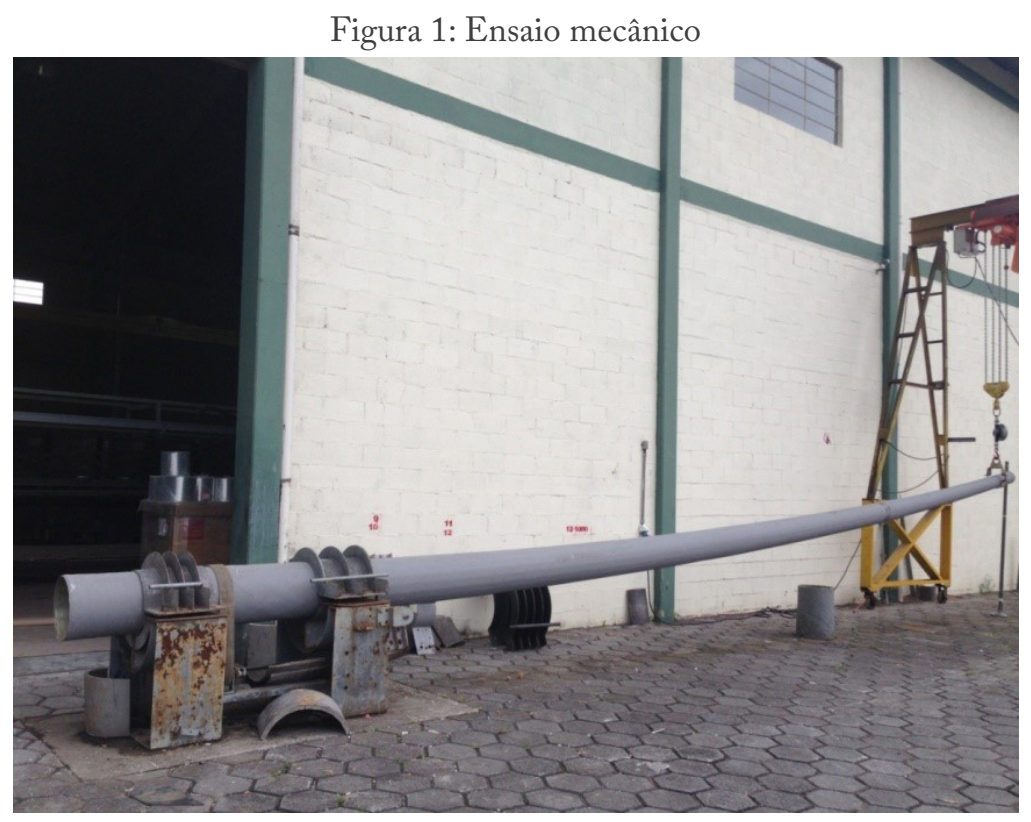

Fonte: TECHNOFIX (2016)

Considerando que a estrutura é oca, ela tende a ovalizar quando submetida a uma força transversal. Quando é aplicada uma força transversal no topo do poste, a geratriz onde a força é aplicada fica tracionada, enquanto a geratriz oposta fica comprimida. $\mathrm{Na}$ região próxima ao engastamento, como a estrutura não sofre deslocamentos, a seção transversal tende a ovalizar, como ilustrado na Figura 2. 


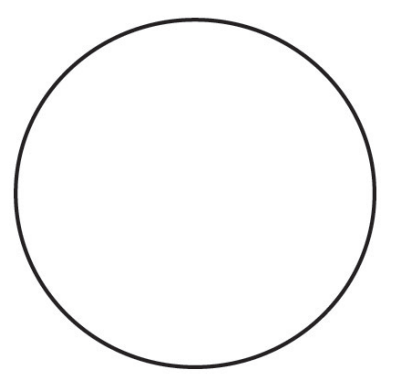

Estrutura indeformada
Tensão resultante da força aplicada

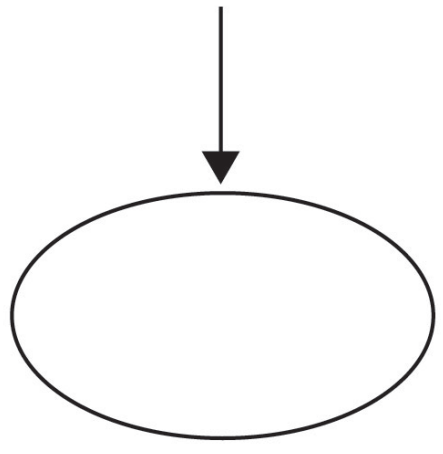

Estrutura submetida à força no topo

Fonte: do autor (2016)

Foi considerado que $1800 \mathrm{~mm}$ do poste estavam enterrados. Esta medida corresponde a $10 \%$ do comprimento do poste mais $600 \mathrm{~mm}$, comprimento de engaste recomendado pela NBR 8451 (ABNT, 2011).

\subsection{ANÁLISE ESTRUTURAL}

A estrutura foi modelada em um programa de análise estrutural através do método dos elementos finitos, utilizando um elemento de casca. As áreas deste elemento foram então divididas na ordem de $10^{\circ}$ cada, totalizando 36 divisões no sentido radial.

No sentido vertical, a divisão da estrutura para análise de deslocamentos se deu da seguinte forma: os três primeiros metros, partindo do fim do engaste, foram divididos em seções de $500 \mathrm{~mm}$. A partir daí, dividiu-se o poste em seis seções de $1 \mathrm{~m}$ cada e uma última seção de 1,20 m.A posição da força e a divisão das seções podem ser observadas na Figura 3.
Figura 3: Representação das divisões da estrutura

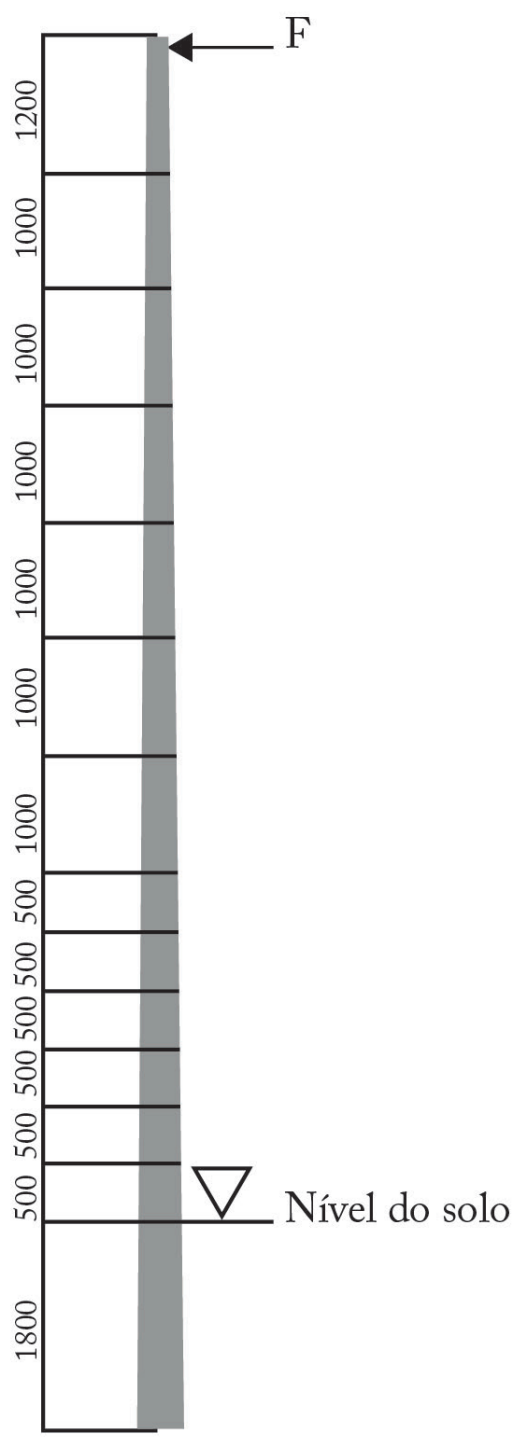

Fonte: do autor (2016) 
Nos dois primeiros metros, o material foi modelado com dois ângulos de enrolamento diferentes, 23 e 90 graus, compondo as camadas conforme citado no item 3.2, enquanto que no restante do poste o ângulo foi considerado constante de 23 graus.

Já para a análise das tensões normais, optou-se por realizar uma divisão mais minuciosa, de forma a propiciar uma melhor precisão. A tensão foi determinada a cada $10 \mathrm{~cm}$, porém apenas nos três primeiros metros, a contar do engastamento.

O carregamento utilizado consistiu em uma força de $50 \mathrm{daN}$ aplicada a $10 \mathrm{~cm}$ do topo da estrutura, aumentando-se o módulo da força em 50 daN até atingir $500 \mathrm{daN}$.

\section{RESULTADOS E ANÁLISES}

Os resultados de deslocamentos e de tensões observados no estudo experimental e na análise computacional da estrutura são apresentados neste tópico.

\subsection{ANÁLISE COMPUTACIONAL DA ESTRUTURA}

Os resultados dos deslocamentos extraídos foram dos pontos das extremidades esquerda e direita, conforme demonstrado na Figura 4, a fim de observar se houve ou não registro de ovalização na estrutura.

Figura 4: Vista em corte da estrutura.

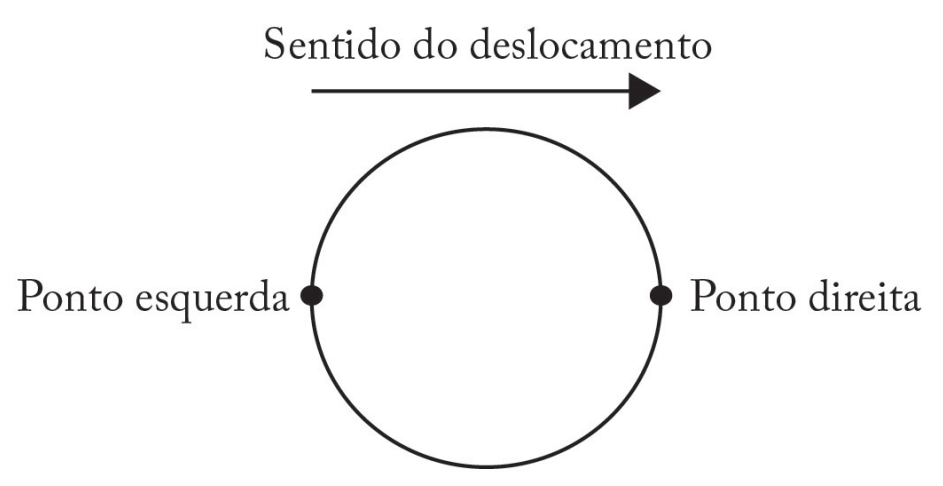

Fonte: do autor (2016)

Foi possível observar que os deslocamentos dos pontos da direita e da esquerda em uma mesma altura são muito próximos, o que significa que o programa não detectou qualquer estrangulamento na estrutura. As curvas de deslocamentos para cada carregamento são apresentadas na Figura 5. 
Figura 5: Curvas de deslocamentos da estrutura obtidas no programa de análise estrutural

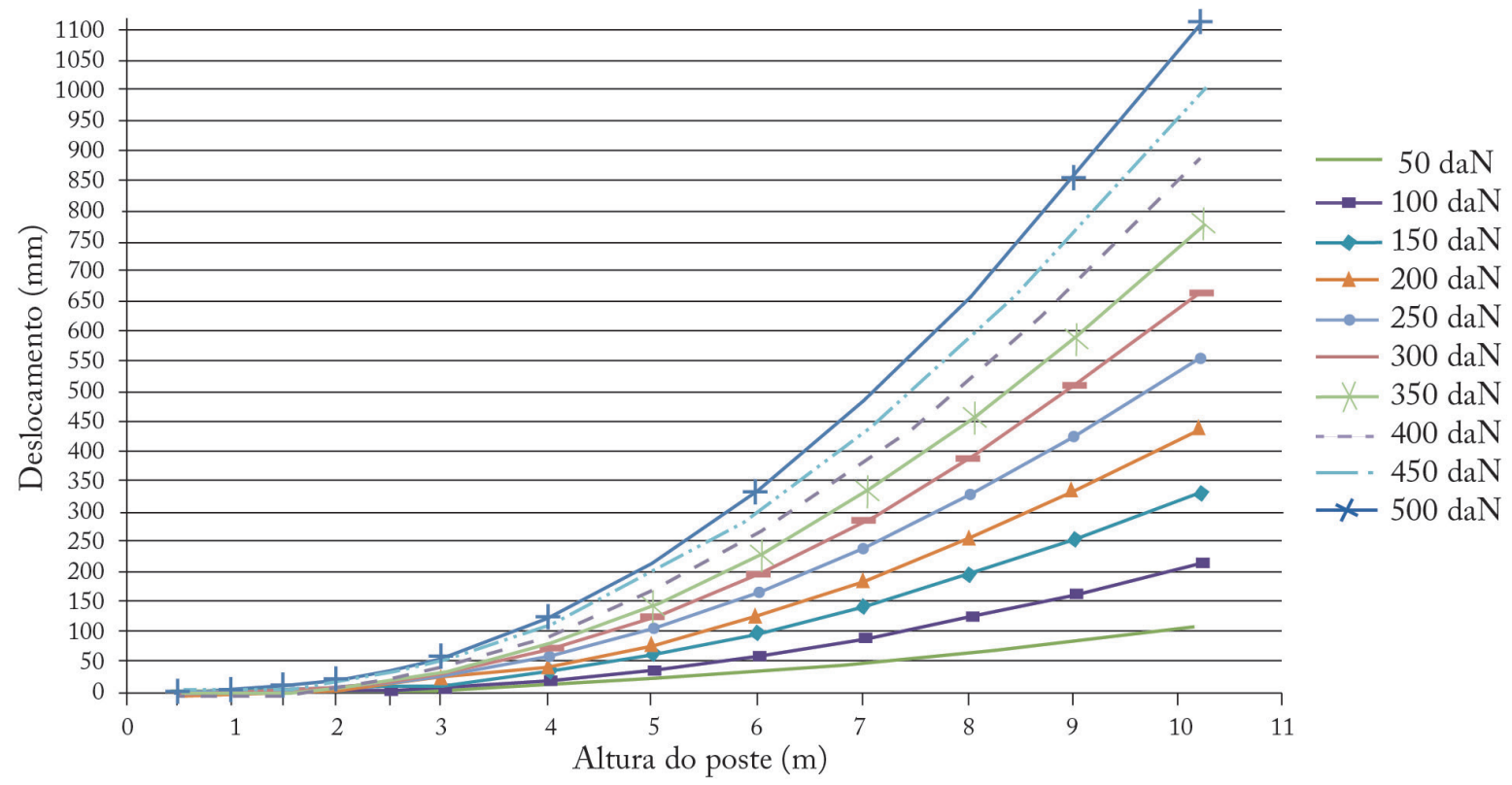

Fonte: do autor (2016)

A determinação das tensões também ocorreu ao longo das geratrizes esquerda e direita da estrutura, como indicado na Figura 3. Os resultados de tensões obtidos na análise para os níveis de carregamento de 200 daN, que corresponde à resistência nominal do poste, e de 500 daN, que corresponde à carga máxima aplicada, são apresentados na Tabela 2.

Tabela 2: Resultados de tensão normal para carga de 200 daN e 500 daN

\begin{tabular}{c|c|c|c|c}
\multirow{2}{*}{ Altura $(\mathbf{m})$} & \multicolumn{2}{|c|}{$\mathbf{2 0 0}$ daN } & \multicolumn{2}{c}{$\mathbf{5 0 0}$ daN } \\
\cline { 2 - 5 } & $\begin{array}{c}\text { Tensão esquerda } \\
(\mathbf{M P a})\end{array}$ & $\begin{array}{c}\text { Tensão direita } \\
\mathbf{( M P a )}\end{array}$ & $\begin{array}{c}\text { Tensão esquerda } \\
(\mathbf{M P a})\end{array}$ & $\begin{array}{c}\text { Tensão direita } \\
(\mathbf{M P a})\end{array}$ \\
\hline 3,0 & 31,7 & $-31,7$ & 79,3 & $-79,3$ \\
\hline 2,9 & 31,8 & $-31,7$ & 79,5 & $-79,4$ \\
\hline 2,8 & 31,8 & $-31,8$ & 79,6 & $-79,5$ \\
\hline 2,7 & 29,7 & $-32,9$ & 79,7 & $-79,6$ \\
\hline 2,6 & 31,9 & $-31,9$ & 79,8 & $-79,7$ \\
\hline 2,5 & 32,0 & $-31,9$ & 79,9 & $-79,8$ \\
\hline 2,4 & 32,0 & $-31,9$ & 79,9 & $-79,9$ \\
\hline
\end{tabular}




\begin{tabular}{|c|c|c|c|c|}
\hline \multirow[b]{2}{*}{ Altura (m) } & \multicolumn{2}{|c|}{$200 \mathrm{daN}$} & \multicolumn{2}{|c|}{$500 \mathrm{daN}$} \\
\hline & $\begin{array}{c}\text { Tensão esquerda } \\
\text { (MPa) }\end{array}$ & $\begin{array}{l}\text { Tensão direita } \\
\text { (MPa) }\end{array}$ & $\begin{array}{c}\text { Tensão esquerda } \\
\text { (MPa) }\end{array}$ & $\begin{array}{c}\text { Tensão direita } \\
\text { (MPa) }\end{array}$ \\
\hline 2,3 & 32,1 & $-32,0$ & 80,2 & $-80,1$ \\
\hline 2,2 & 34,0 & $-34,0$ & 85,1 & $-85,0$ \\
\hline 2,1 & 34,2 & $-34,2$ & 85,5 & $-85,6$ \\
\hline 2,0 & 34,6 & $-34,5$ & 86,5 & $-86,3$ \\
\hline 1,9 & 17,2 & $-17,2$ & 42,9 & $-43,0$ \\
\hline 1,8 & 17,1 & $-17,1$ & 42,7 & $-42,7$ \\
\hline 1,7 & 17,0 & $-17,1$ & 42,5 & $-42,7$ \\
\hline 1,6 & 16,9 & $-17,1$ & 42,3 & $-42,4$ \\
\hline 1,5 & 16,9 & $-17,1$ & 42,3 & $-42,8$ \\
\hline 1,4 & 16,9 & $-17,0$ & 42,3 & $-42,5$ \\
\hline 1,3 & 16,8 & $-17,0$ & 42,0 & $-42,7$ \\
\hline 1,2 & 16,8 & $-17,0$ & 42,2 & $-42,4$ \\
\hline 1,1 & 16,7 & $-17,0$ & 41,7 & $-42,6$ \\
\hline 1,0 & 17,0 & $-16,8$ & 42,4 & $-42,0$ \\
\hline 0,9 & 16,9 & $-17,2$ & 42,2 & $-43,0$ \\
\hline 0,8 & 17,2 & $-15,8$ & 42,9 & $-39,4$ \\
\hline 0,7 & 17,1 & $-15,8$ & 42,9 & $-39,4$ \\
\hline 0,6 & 17,1 & $-15,7$ & 42,8 & $-39,4$ \\
\hline 0,5 & 17,2 & $-17,6$ & 43,0 & $-44,0$ \\
\hline 0,4 & 16,7 & $-16,7$ & 41,8 & $-41,8$ \\
\hline 0,3 & 17,5 & $-17,8$ & 43,7 & $-44,5$ \\
\hline 0,2 & 16,8 & $-17,0$ & 41,9 & $-42,4$ \\
\hline 0,1 & 20,4 & $-20,9$ & 51,0 & 52,1 \\
\hline
\end{tabular}

Fonte: do autor (2016)

Assim como os valores de deslocamento, as tensões de tração e compressão de mesma altura possuem módulos parecidos, o que não demonstra a existência de deformações da seção transversal da estrutura. 


\subsection{ENSAIO DE CAMPO DA ESTRUTURA}

Para que fosse possível estabelecer uma correlação entre a análise realizada no modelo computacional de cálculo e a estrutura em situação de utilização, foi realizado o ensaio de campo baseado no método proposto pela NBR 8451 (ABNT, 2011).

O poste foi posicionado na bancada e travado até o comprimento de $1800 \mathrm{~mm}$, conforme indicado na Figura 6.

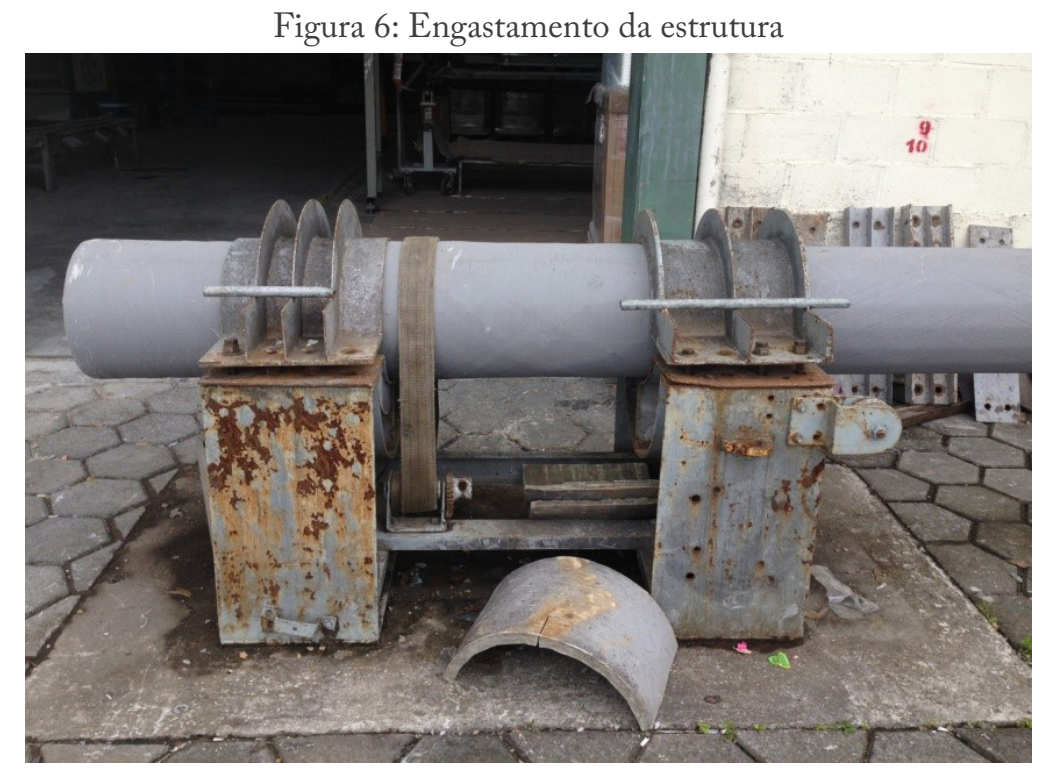

Fonte: TECHNOFIX (2016)

Foram efetuadas medidas verticais do diâmetro do poste em todas as seções de análise a cada aumento de força. Após as medições para o nível de carregamento solicitado em norma, o poste foi submetido ao carregamento até a ruptura, que ocorreu com uma carga de 550 $\mathrm{daN}$, a $2 \mathrm{~m}$ acima do ponto de engastamento, na face comprimida. A estrutura rompida pode ser observada na Figura 7.

\section{Figura 7: Estrutura rompida}

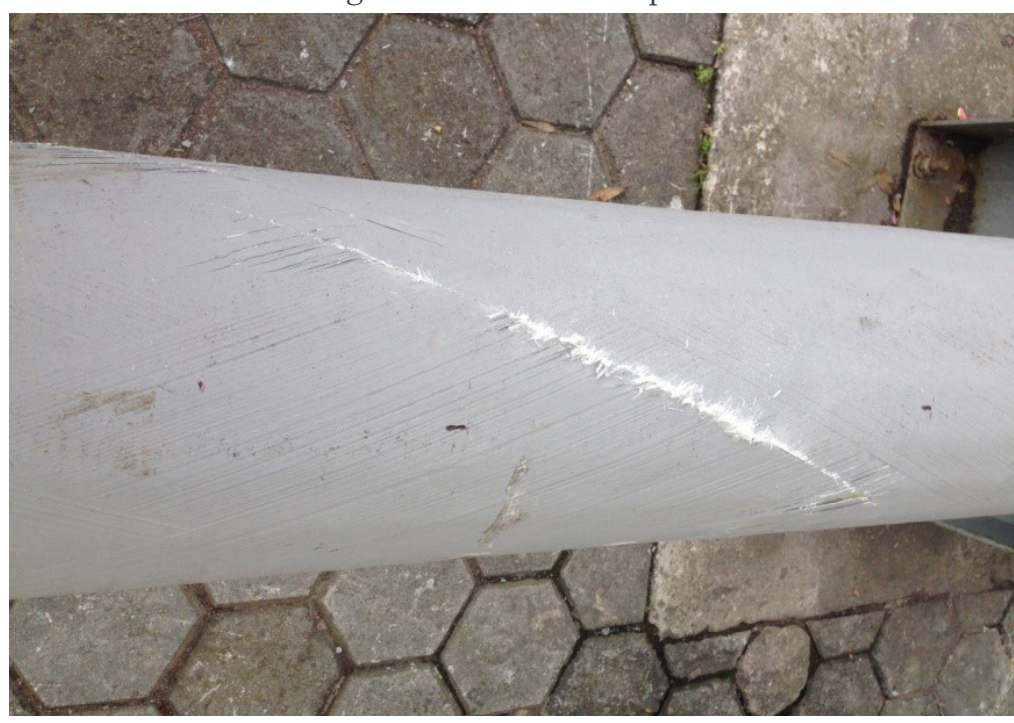

Fonte: TECHNOFIX (2016) 
Os resultados de variação do diâmetro e deslocamento do topo do poste, obtidos no ensaio de campo, estão apresentados nas Tabelas 3 e 4:

Tabela 3: Diâmetros medidos no ensaio de campo (mm)

\begin{tabular}{|c|c|c|c|c|c|c|c|c|c|c|c|}
\hline \multirow{2}{*}{$\begin{array}{c}\text { Altu- } \\
\text { ra }\end{array}$} & \multicolumn{11}{|c|}{ Diâmetros (mm) } \\
\hline & 0 & 50 & 100 & 150 & 200 & 250 & 300 & 350 & 400 & 450 & 500 \\
\hline 9,0 & 171,2 & 171,2 & 171,2 & 171,2 & 170,2 & 170,2 & 170,2 & 170,2 & 170,2 & 170,2 & 170,2 \\
\hline 8,0 & 193,2 & 192,2 & 192,2 & 192,2 & 192,2 & 192,2 & 192,2 & 192,2 & 192,2 & 192,2 & 192,2 \\
\hline 7,0 & 212,7 & 212,7 & 212,7 & 212,7 & 212,2 & 212,2 & 212,2 & 212,2 & 212,2 & 212,2 & 212,2 \\
\hline 6,0 & 239,2 & 239,2 & 239,2 & 239,2 & 238,2 & 238,2 & 238,2 & 238,2 & 238,2 & 238,2 & 238,2 \\
\hline 5,0 & 258,2 & 258,2 & 258,2 & 258,2 & 258,2 & 258,2 & 257,7 & 257,7 & 256,2 & 256,2 & 256,2 \\
\hline 4,0 & 282,2 & 280,2 & 280,2 & 280,2 & 280,2 & 280,2 & 280,2 & 280,2 & 279,7 & 278,7 & 278,7 \\
\hline 3,0 & 298,2 & 298,2 & 298,2 & 298,2 & 297,2 & 297,2 & 295,7 & 294,2 & 294,2 & 292,2 & 290,2 \\
\hline 2,5 & 314,7 & 314,7 & 314,7 & 314,7 & 314,7 & 313,2 & 311,7 & 310,2 & 308,2 & 303,2 & 303,7 \\
\hline 2,0 & 324,7 & 323,2 & 323,2 & 323,2 & 322,2 & 321,7 & 320,7 & 317,7 & 315,2 & 312,2 & 322,7 \\
\hline 1,5 & 333,2 & 333,2 & 333,2 & 333,2 & 333,2 & 330,7 & 330,2 & 327,2 & 325,7 & 324,2 & 322,7 \\
\hline 1,0 & 343,2 & 343,2 & 343,2 & 343,2 & 342,2 & 341,7 & 341,2 & 338,2 & 337,7 & 335,7 & 334,7 \\
\hline 0,5 & 350,2 & 350,2 & 350,2 & 350,2 & 350,2 & 349,2 & 348,2 & 346,2 & 346,2 & 346,2 & 344,7 \\
\hline
\end{tabular}

Fonte: do autor (2016)

Tabela 4: Deslocamento no topo

\begin{tabular}{l|c|c|c|c|c|c|c|c|c|c}
\hline Força (daN) & 50 & 100 & 150 & 200 & 250 & 300 & 350 & 400 & 450 & 500 \\
\hline Deslocamento de campo $(\mathrm{mm})$ & 115 & 224 & 343 & 465 & 593 & 742 & 870 & 1010 & - & - \\
\hline
\end{tabular}

Fonte: do autor (2016)

Não foi possível medir o deslocamento no topo da estrutura para as forças de 450 e $500 \mathrm{daN}$, devido à limitação do equipamento de medição, pois a deformação ultrapassou o limite do equipamento.

\subsection{COMPARAÇÃO DE RESULTADOS}

$\mathrm{Na}$ tabela 5, estão apresentados os valores dos deslocamentos no topo do poste obtidos experimentalmente e os valores extraídos do modelo 
matemático. Para esta análise, foram consideradas as medidas dos pontos da face esquerda, pois é a que corresponde à medida realizada no ensaio mecânico.

Tabela 5: Comparação entre resultados de deslocamento

\begin{tabular}{l|c|c|c|c|c|c|c|c|c|c}
\hline Força (daN) & 50 & 100 & 150 & 200 & 250 & 300 & 350 & 400 & 450 & 500 \\
\hline Deslocamento de campo (mm) & 112 & 224 & 336 & 447 & 559 & 671 & 783 & 895 & 1007 & 1119 \\
\hline Deslocamento de campo (mm) & 115 & 224 & 343 & 465 & 593 & 742 & 870 & 1010 & - & - \\
\hline Diferença (m m) & 3 & 0 & 7 & 18 & 34 & 71 & 87 & 115 & - & - \\
\hline Diferença (\%) & 2,61 & 0,00 & 2,04 & 3,87 & 5,73 & 9,57 & 10,00 & 11,39 & - & - \\
\hline
\end{tabular}

Fonte: do autor (2016)

É possível observar que, até o valor de $200 \mathrm{daN}$, correspondente à resistência nominal do poste, a variação entre a medida obtida experimentalmente e a medida teórica é inferior a $4 \%$. No entanto, entre a carga de $250 \mathrm{daN}$ e 300 daN houve uma modificação na tendência de crescimento da diferença. Também é possível constatar que o deslocamento do topo da estrutura se manteve dentro do limite estabelecido pela concessionária de energia até o valor de carga correspondente à resistência nominal do poste, que é de $4 \%$ do comprimento do poste, ou seja, $480 \mathrm{~mm}$.

Quanto à ovalização, o programa de modelagem não registrou valores significativos de estrangulamento no diâmetro da estrutura. A diferença entre as medidas dos deslocamentos dos lados esquerdo e direito de mesma altura do programa extraídas de análise podem ser observadas na Tabela 6.

Tabela 6: Diferença entre deslocamentos obtidos no programa computacional ( $\mathrm{mm}$ )

\begin{tabular}{c|c|c|c|c|c|c|c|c|c|c}
\hline \multirow{2}{*}{ Altura } & \multicolumn{7}{c}{ Carga de ensaio (daN) } \\
\cline { 2 - 11 } & 50 & 100 & 150 & 200 & 250 & 300 & 350 & 400 & 450 & 500 \\
\hline 9,0 & 0,00 & 0,00 & 0,00 & 0,00 & 0,00 & 0,00 & 0,00 & 0,00 & 0,00 & 0,00 \\
\hline 8,0 & 0,00 & 0,00 & 0,00 & 0,00 & 0,00 & $\mathbf{0 , 0 0}$ & $\mathbf{0 , 0 0}$ & $\mathbf{0 , 0 0}$ & $\mathbf{0 , 0 0}$ & $\mathbf{0 , 0 0}$ \\
\hline 7,0 & $\mathbf{0 , 0 0}$ & $\mathbf{0 , 0 0}$ & $\mathbf{0 , 0 0}$ & $\mathbf{0 , 0 0}$ & $\mathbf{0 , 0 0}$ & $\mathbf{0 , 0 0}$ & $\mathbf{0 , 0 0}$ & $\mathbf{0 , 0 0}$ & $\mathbf{0 , 0 0}$ & $\mathbf{0 , 0 0}$ \\
\hline $\mathbf{6 , 0}$ & $\mathbf{0 , 0 0}$ & $\mathbf{0 , 0 0}$ & $\mathbf{0 , 0 0}$ & $\mathbf{0 , 0 0}$ & $\mathbf{0 , 0 0}$ & $\mathbf{0 , 0 0}$ & $\mathbf{0 , 0 0}$ & $\mathbf{0 , 0 0}$ & $\mathbf{0 , 0 0}$ & $\mathbf{0 , 0 0}$ \\
\hline $\mathbf{5 , 0}$ & $\mathbf{0 , 0 0}$ & $\mathbf{0 , 0 0}$ & $\mathbf{0 , 0 0}$ & $\mathbf{0 , 0 0}$ & $\mathbf{0 , 0 0}$ & $\mathbf{0 , 0 0}$ & $\mathbf{0 , 0 0}$ & $\mathbf{0 , 0 0}$ & $\mathbf{0 , 0 0}$ & $\mathbf{0 , 0 0}$ \\
\hline 4,0 & $\mathbf{0 , 0 0}$ & $\mathbf{0 , 0 0}$ & $\mathbf{0 , 0 0}$ & $\mathbf{0 , 0 0}$ & $\mathbf{0 , 0 0}$ & $\mathbf{0 , 0 0}$ & $\mathbf{0 , 0 0}$ & $\mathbf{0 , 0 0}$ & $\mathbf{0 , 0 0}$ & $\mathbf{0 , 0 0}$ \\
\hline
\end{tabular}




\begin{tabular}{c|c|c|c|c|c|c|c|c|c|c}
\hline \multirow{2}{*}{ Altura } & \multicolumn{7}{|c}{ Carga de ensaio (daN) } \\
\cline { 2 - 11 } & 50 & 100 & 150 & 200 & 250 & 300 & 350 & 400 & 450 & 500 \\
\hline 3,0 & 0,00 & 0,00 & 0,00 & 0,00 & 0,00 & 0,00 & 0,00 & $-0,01$ & $-0,01$ & $-0,01$ \\
\hline 2,5 & 0,00 & $-0,01$ & $-0,01$ & $-0,01$ & $-0,01$ & $-0,02$ & $-0,02$ & $-0,02$ & $-0,02$ & $-0,03$ \\
\hline 2,0 & $-0,01$ & $-0,01$ & $-0,02$ & $-0,03$ & $-0,03$ & $-0,04$ & $-0,04$ & $-0,05$ & $-0,05$ & $-0,06$ \\
\hline 1,5 & $-0,01$ & $-0,01$ & $-0,02$ & $-0,02$ & $-0,03$ & $-0,03$ & $-0,04$ & $-0,04$ & $-0,04$ & $-0,05$ \\
\hline 1,0 & $-0,01$ & $-0,01$ & $-0,02$ & $-0,02$ & $-0,03$ & $-0,03$ & $-0,04$ & $-0,04$ & $-0,04$ & $-0,05$ \\
\hline 0,5 & $-0,01$ & $-0,02$ & $-0,03$ & $-0,03$ & $-0,04$ & $-0,05$ & $-0,06$ & $-0,07$ & $-0,07$ & $-0,08$ \\
\hline
\end{tabular}

Fonte: do autor (2016)

É possível observar uma pequena ovalização entre $1,5 \mathrm{~m}$ e 2,5 $\mathrm{m}$, porém os valores obtidos são inferiores aos valores medidos no ensaio mecânico realizado em campo, conforme apresentado na Tabela 7, na qual é possível observar o estrangulamento da estrutura, medido pela diminuição do diâmetro, conforme o acréscimo da força aplicada na estrutura.

Tabela 7: Variação do diâmetro (mm)

\begin{tabular}{c|c|c|c|c|c|c|c|c|c|c}
\multirow{2}{*}{ Altura } & \multicolumn{7}{c}{ Carga de ensaio (daN) } \\
\cline { 2 - 11 } & 50 & 100 & 150 & 200 & 250 & 300 & 350 & 400 & 450 & 500 \\
\hline 9,0 & 0,0 & 0,0 & 0,0 & $-1,0$ & $-1,0$ & $-1,0$ & $-1,0$ & $-1,0$ & $-1,0$ & $-1,0$ \\
\hline 8,0 & $-1,0$ & $-1,0$ & $-1,0$ & $-1,0$ & $-1,0$ & $-1,0$ & $-1,0$ & $-1,0$ & $-1,0$ & $-1,0$ \\
\hline 7,0 & 0,0 & 0,0 & 0,0 & $-0,5$ & $-0,5$ & $-0,5$ & $-0,5$ & $-0,5$ & $-0,5$ & $-0,5$ \\
\hline 6,0 & 0,0 & 0,0 & 0,0 & $-1,0$ & $-1,0$ & $-1,0$ & $-1,0$ & $-1,0$ & $-1,0$ & $-1,0$ \\
\hline 4,0 & 0,0 & 0,0 & 0,0 & 0,0 & 0,0 & $-0,5$ & $-0,5$ & $-2,0$ & $-2,0$ & $-2,0$ \\
\hline 3,0 & $-2,0$ & $-2,0$ & $-2,0$ & $-2,0$ & $-2,0$ & $-2,0$ & $-2,0$ & $-2,5$ & $-3,5$ & $-3,5$ \\
\hline 2,5 & 0,0 & 0,0 & 0,0 & $-1,0$ & $-1,0$ & $-2,5$ & $-4,0$ & $-4,0$ & $-6,0$ & $-8,0$ \\
\hline 2,0 & 0,0 & 0,0 & 0,0 & 0,0 & $-1,5$ & $-3,0$ & $-4,5$ & $-6,5$ & $-11,5$ & $-11,0$ \\
\hline & $-1,5$ & $-1,5$ & $-1,5$ & $-2,5$ & $-3,0$ & $-4,0$ & $-7,0$ & $-9,5$ & $-12,5$ & $-13,5$ \\
\hline
\end{tabular}




\begin{tabular}{c|c|c|c|c|c|c|c|c|c|c}
\hline \multirow{2}{*}{ Altura } & \multicolumn{10}{c}{ Carga de ensaio (daN) } \\
\cline { 2 - 11 } & 50 & 100 & 150 & 200 & 250 & 300 & 350 & 400 & 450 & 500 \\
\hline 1,5 & 0,0 & 0,0 & 0,0 & 0,0 & $-2,5$ & $-3,0$ & $-6,0$ & $-7,5$ & $-9,0$ & $-10,5$ \\
\hline 1,0 & 0,0 & 0,0 & 0,0 & $-1,0$ & $-1,5$ & $-2,0$ & $-5,0$ & $-5,5$ & $-7,5$ & $-8,5$ \\
\hline 0,5 & 0,0 & 0,0 & 0,0 & 0,0 & $-1,0$ & $-2,0$ & $-4,0$ & $-4,0$ & $-4,0$ & $-5,5$ \\
\hline
\end{tabular}

Fonte: do autor (2016)

A partir da Tabela 7, estabeleceu-se a curva de ovalização da estrutura solicitada a 500 daN.No mesmo gráfico, sobrepôs-se a curva de tensões de compressão, obtida no modelo matemático, da estrutura quando solicitada a mesma carga. Esta correlação entre ovalização e tensão pode ser observada na Figura 8.

Figura 8: Correlação entre ovalização e tensão

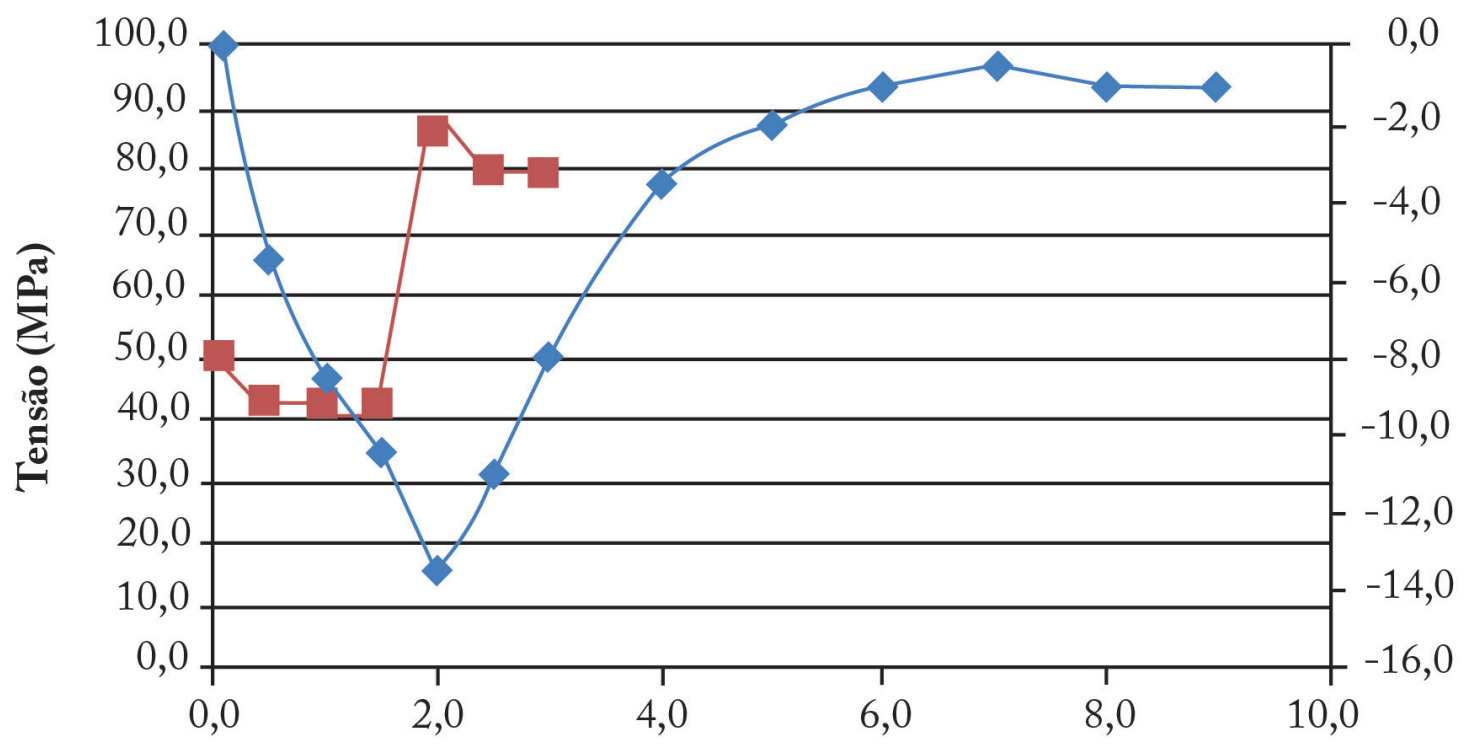

Altura do poste (m)

—Tensão —Ovalização

Fonte: do autor (2016)

Observando a Tabela 7 e a Figura 8, constata-se que a maior ovalização sofrida foi na altura de 2 $\mathrm{m}$, a partir do engastamento, em que o diâmetro do poste diminuiu 4,16\% em relação ao seu valor inicial, assim como as maiores tensões também ocorreram nesta região. No ensaio de campo, foi verificado que o poste rompeu na face comprimida, na direção das fibras, na altura de $2 \mathrm{~m}$. Esta altura corresponde exatamente ao fim da camada de $90^{\circ}$ das fibras. Pelos resultados apresentados, acredita-se que esta camada contribuiu para o aumento da rigidez, pois diminuiu a ovalização, e também para uma melhoria da resistência, pois as tensões atuantes passaram de valores próximos de $40 \mathrm{MPa}$ para valores da ordem de $80 \mathrm{MPa}$. 


\section{CONCLUSÕES}

$\mathrm{Na}$ análise dos deslocamentos, a modelagem utilizada apresentou resultados compatíveis com os resultados dos ensaios realizados em campo até o valor da resistência nominal do poste. Para os valores de carga superiores, foi verificado um deslocamento maior no ensaio de campo, que pode ter sido motivado pela possível ocorrência de microfissuras na estrutura, o que provocou alteração na curva elástica do material. O método de análise estrutural considera o material perfeitamente elástico, e não considera a seção fissurada, sendo que o aparecimento de fissuras altera o comportamento do material. Outro fator que pode ter influenciado nesta divergência de resultados seria um comportamento plástico do material nos ensaios de campo, para níveis mais elevados de carregamento, fato este não considerado nas análises de deslocamento e computacional. Também tem que se considerar o engaste do material, que, para pequenas cargas, a estrutura se comportou praticamente como perfeitamente engastada, correspondendo ao que foi considerado na modelagem computacional. Porém, para cargas mais elevadas, pode ter ocorrido algum deslocamento nos parafusos e/ou nas chapas metálicas que realizavam o travamento da estrutura, propiciando maior deslocamento no topo da mesma. Ainda existe a possibilidade de que esta diferença tenha ocorrido devido à anisotropia do material, que na modelagem computacional não foi considerada, tendo em vista ainda que, durante o ensaio, na região tracionada do poste, a carga era transferida para as fibras e, na região comprimida, para a matriz, elementos que possuem propriedades diferentes.

Para a avaliação da ovalização da estrutura, a modelagem não pode ser considerada, uma vez que não se conseguiu reproduzir as deformações observadas no ensaio de campo.A única maneira de relacionar a ovalização registrada no ensaio de campo com o modelo computacional é pela análise de tensões, na qual foram registradas as maiores tensões exatamente nolocal onde ocorreu a maior ovalização e a ruptura da estrutura. Portanto, é possível considerar que o local em que ocorrerá a maior ovalização será onde estão indicadas as maiores tensões. Um aspecto que pode ter influenciado na análise computacional consiste no fato de que a ovalização produz uma redução no momento de inércia da peça, diminuindo a sua rigidez e aumentando os deslocamentos.

\section{AGRADECIMENTOS}

Agradecemos à empresa TECHNOFIX Ind. E Com. De Fibra de Vidro LTDA, pelo total apoio ao presente trabalho. 


\section{REFERÊNCIAS}

\section{ASSOCIAÇÃO BRASILEIRA DE}

MATERIAIS COMPÓSITOS - ABMACO.

Compósitos I: Materiais, Processos, Aplicações, Desempenhos e Tendências. 2008.

ASSOCIAÇÃO BRASILEIRA DE NORMAS TÉCNICAS. NBR 8451: postes de concreto armado e protendido para redes de distribuição e de transmissão de energia elétrica. Rio de Janeiro, 2011.

ALVES, Lucas Máximo. Método dos Elementos Finitos. Dissertação - Universidade Federal do Paraná. Curitiba: UFPR, 2007.

AZEVEDO, Álvaro F. M. Método dos Elementos Finitos. 2003. Faculdade de Engenharia da Universidade do Porto. Portugal: FEUP, 2003.

CALLISTER Jr., William D. Ciência e

Engenharia de Materiais: uma introdução. Rio de Janeiro: LTC, 2002.

FARIA, Paulo Eustáquio de. Estudo Sobre a Furação de Compósito de Resina Epóxi Reforçado com Fibras de Vidro. 2007.231. Tese - Universidade Federal de Minas Gerais. Belo Horizonte: UFMG, 2007.

FERREIRA, José Carlos Lopes da Rocha. Postes Estruturais em Sistemas Compósitos. 2009. Tese - Universidade do Porto. Porto: UP, 2009.

FORTE, Marco Aurélio. Plásticos Reforçados com fibra de vidro. COOPMACO, 2014.

GESUALDO, Francisco A. R. Método dos Elementos Finitos. 2010. 53. Dissertação Universidade Federal de Uberlândia. Uberlândia: UFU, 2010.

MARINUCCI, Gerson. Materiais Compósitos Poliméricos. São Paulo: Artliber, 2011.

ORTENZI Jr., Altibano. A Fibra de Vidro em Matrizes Poliméricas e Cimentícias e Seu Uso Estrutural Em Construção Civil. 2007. Dissertação, Universidade Federal de São Carlos. São Carlos: UFSCAR, 2007.

ZURSTRASSEN E. Materiais 2: reforços. In: ASSOCIAÇÃO BRASILEIRA DOS MATERIAIS COMPÓSITOS - ABMACO. Compósitos I. São Paulo: ABMACO, 2008.

\section{SOBRE OS AUTORES}

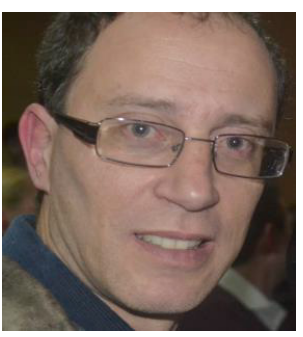

Wellington Mazer

Possui graduação em Engenharia Civil pela Universidade Federal do Paraná (1994), especialização em Construção Civil Estrutura (1996), mestrado em Engenharia Hidráulica pela Universidade Federal do Paraná (2003) e doutorado em Infraestrutura Aeronáutica pelo ITA.Atualmente é professor do Ensino Básico, Técnico e Tecnológico da Universidade Tecnológica Federal do Paraná, ministrando aulas nos cursos de Engenharia Civil, Arquitetura e Urbanismo e na Pós-graduação em Patologia das Construções, nas disciplinas de Argamassas e Concretos, Concretos Especiais, Patologia das Construções e Sistemas Estruturais. Tem experiência na área de Engenharia Civil, com ênfase em Estruturas de Concreto, atuando principalmente nos seguinte temas: concreto, durabilidade, estruturas, patologia do concreto e dosagem de concretos.

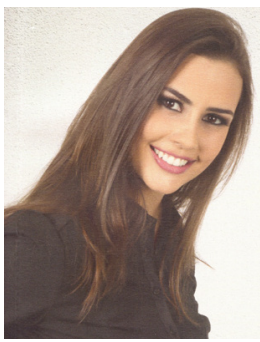

\section{Maria Luiza Ribas Christo}

Engenheira civil graduada pela Universidade Tecnológica Federal do Paraná. Um ano de graduação cursado na Alemanha, em Bauhaus Universität Weimar.Já atuou na construção civil, em obras residenciais. Atualmente atua como engenheira civil no ramo de produção de postes e cruzetas de poliéster reforçado com fibra de vidro (PRFV). 